\title{
Für immer Dein?
}

\author{
Postsystematische Erwägungen zur Wahrnehmung der \\ Ewigkeit auf der christlichen Weglinienperspektive
}

Markus Mühling

\section{Das pseudosäkulare Narrativ von »Für-immer- Erzählungen«}

\section{$1.1 » F u ̈ r$ immer Dein«}

"Für immer Dein" ist nicht nur eine äußerst zweischneidige Verheißung, die - vom Stalker ausgesprochen - sofort zum Gericht mit Verdammungsandrohung wird, sondern auch hypernervöse und übersteigerte säkulare Theologie. Wie brutal nüchtern wirkt das »bis dass der Tod Euch scheidet « der kirchlichen Trauung doch gegen den Liebesschwur "für immer Dein". Im Englischen scheint "forever« allerdings manchmal nicht auszureichen. Die biblische Übersetzung in liturgischen und säkularen Kontexten lautet: "forever and ever.$^{1}$ Das kann leicht missverstanden werden, so dass sich hier ein trivialisierter Titanismus spiegeln mag: Offensichtlich bedarf auch das »Für-Immer» noch einer Steigerung, so als würde es ohne diese entgleiten. Logisch gesehen ist "für immer" hingegen ein Konzept zweiter Ordnung, das für sich genommen leer ist und erst bedeutsam wird, wenn geklärt ist, auf welche Ereignisse, Qualitäten oder Prädikate es sich beziehen soll. Im Falle des »Für-Immer-Dein« ist dies die romantische Liebe des gelebten Popsongs des impliziten "amerikanischen Credos des Romantizismus" und damit wiederum ein unscharfer Begriff, unter dem man sich alles Mögliche vorstellen mag. ${ }^{2}$ Das aber macht die manipulative Wirkmächtigkeit des »Für-

\footnotetext{
1 „Forever and ever" ist übliche Übersetzung des griechischen "

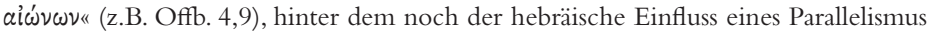
membrorum (לעולם ועד) erkennbar ist.

2 Vgl. Morton M. Hunt, The Natural History of Love, Scranton 1959, 363-367, und zur weiteren Analyse Dwight van de Vate Jr., Romantic Love. A Philosophical Inquiry, London 1981, 52.
} 
Immer-Dein" gerade aus: Je weniger der Verheißende verspricht, desto mehr und vor allem desto mehr eigene Wünsche mag der Verheißungsempfänger in das Versprechen hineinlegen. Sei es, dass eigene Wünsche übersteigert, eigene Befürchtungen negiert oder sich ein Lebensglück erhofft wird, dessen Urheber der Partner ist ${ }^{3}$ : Beim »Für-Immer" handelt es sich um eine snatürliche Theologie der Ewigkeit oder eine `natürliche Theologie der Liebe`, die die klassischen Wege der Eigenschaftserkenntnis Gottes - via gloriae, via negationis, via causalitatis - nachahmt und in ihrer Rigorosität rechts überholt. Der Verheißungsempfänger wird zum wahren Schöpfer des Verheißungsinhalts, wie eine radikale natürliche Theologie den Menschen an die Stelle Gottes hebt. Der Unterschied mag nur darin bestehen, dass der "Für-immer-Dein-Versprechende" genau dies beabsichtigt: Denn in der Regel handelt es sich dann weniger um einen kommissiven Sprechakt als um den Versuch, den Angesprochenen über einen versteckten Appell an alle narzisstischen, also alle sündhaften Tendenzen zu bezirzen (und Kirke hat ja ihre eigenen Ziele). Auch damit verspricht der "Für-immer-Dein-Verheißende» etwas: Er ahmt letztlich das Versprechen der Schlange »eritis sicut deus « - "Ihr werdet sein wie Gott, indem ihr selbst der Grund der Unterscheidung von Gut und Böse werdet " - nach: "für immer Dein«, also: "Wen immer Du Dir erträumst, ich werde es sein!« Der Versprechende bietet sich also an, genau derjenige sein zu wollen, der stets alle im Auge des Versprechensempfängers ıguten Bedürfnisse erfüllen wird. Zwar sind wir in der Tat Mängelwesen und können Bedürfnisse äußern. Und in der Tat benötigen wir zu deren Erfüllung immer die Hingabe anderer Menschen. Aber: Wenn ich mir zutraue, selbst bestimmen zu können, welche meiner Wünsche und Bedürfnisse wirklich die meinen sind, d.h. welche davon wirklich gut und nicht schlecht sind; dann operiere ich aufgrund der Sünde, weil ich mir eine Definitionsmacht über sgutı und ‘böse` zutraue, die mir nicht zukommt. »Für immer Dein« sagt mir dann zweierlei: 1. »Ja, Du darfst selbst entscheiden, was wirklich gut für Dich ist." 2. »Ich stelle mein ganzes personales Werden Dir in Hingabe so zur Verfügung, dass es all diese Deine Wünsche befriedigt. "Nicht nur die Ver-rücktheit des Versprechensempfängers wird dann deutlich, sondern auch die des Versprechenden. Denn dieser verspricht ja sich selbst - d.h. sein ganzes personales Werden - für den Empfänger zur Verfügung zu stellen. Darüber kann aber keine geschaffene Person

\footnotetext{
3 Vgl. zur Übertragung von Glückshoffnungen auf den romantischen Partner die schöne Analyse in Ulrich Beck/Elisabeth Beck-Gernsheim, Das ganz normale Chaos der Liebe, Frankfurt a.M. 1990, 222-266 (Kap. "Die irdische Religion der Liebe«).
} 
selbst verfügen. Im Klartext gesprochen wird der Betrugsversuch deutlich - und daher darf er eben nicht im Klartext gesprochen werden.

\section{2 »Augenblick, verweile doch!»}

Versuchungen sind nur wirksam, wenn sie nicht als solche erscheinen, sondern Wünsche und Bedürfnisse bedienen, und so gut erscheinen ohne es zu sein. Als Konzept zweiter Ordnung hat es das "Fürimmer-Dein-Versprechen" insofern einfach, weil es sich auf alle unterschiedlichen Hoffnungen und Erwartungen erster Ordnung des Verheißungsempfängers beziehen kann. Aber offensichtlich bedient auch dieVerheißung »für immer" selbst einen eigenen Wunsch, nicht einen erster, sondern einen zweiter Ordnung ${ }^{4}$, und steigert damit die Wirkung des Versprechens bzw. ihrer Verführungskraft: derjenige Wunsch, der im "Augenblick, verweile doch!« zum Ausdruck kommt. Natürlich behandelt Goethe diesen Wunsch verewigter Gegenwart kritisch, kritischer jedenfalls als die trivialisierte Populärversion, die aus dem Wunsch "Augenblick, verweile doch!« das carpe diem macht. Obwohl man vermutlich Horaz nie gelesen hat, wird doch auch hier mit den einschlägigen Übersetzungen "Pflücke den Tag" oder "Genieße den Augenblick" nicht mehr deutlich, dass ebenso korrekte Übersetzungen auch lauten könnten, "Lasset uns essen und trinken, denn morgen werden wir tot sein" $(1$ Kor 15,32) oder »Friß und stirb!« Hedonistische Schmerzverminderung oder Lusterfahrung verliert angesichts der Endlichkeitserfahrung des Menschen offensichtlich viel von ihrem Zauber; ein Zauber freilich, der angesichts der Resonanz des Wunsches "Augenblick, verweile doch!« und des Versprechens "für immer Dein" trotz des Bewusstseins aller Gefahr zwar nicht "für immer", aber immerhin "immer wieder" gewahrt werden kann. Schließlich äußert Faust am Ende von Faust II ja die todbringenden Worte "Verweile doch" - und findet doch gegen die Logik Mephistos nicht den Untergang, sondern gnadenhaft die Rettung.

Noch Vieles ließe sich über die Struktur säkularer »Für-immerErzählungen"sagen - z.B. dass diese offensichtlich contra intentionem doch einen sequenziellen Ablauf, eine Gefährdung der Liebe, Antagonisten, Opfer und die Inklusion des Todes brauchen, sei es die des Antagonisten oder der Protagonisten im Liebestod. Aber hier kann

4 Zur Unterscheidung von first order und second order volitions vgl. Harry G. Frankfurt, Willensfreiheit und der Begriff der Person, in: Analytische Philosophie des Geistes, hg. v. Peter Bieri, Weinheim u.a. 1981, 287-302. 
nicht der Raum sein, dieses ja letztlich jedem bekannte Narrativ im Detail zu beschreiben. Wichtig ist vielmehr, dass die Logik einiger zentraler Bestandteile des Narrativs auch in theologischen Ewigkeitsreflexionen wiederkehren.

\section{Theologische Ewigkeitsreflexionen}

Klassisch-theologische Ewigkeitsreflexionen beziehen zumeist Ewigkeit und Zeitlichkeit aufeinander, und zwar so, dass eine natürliche Theologie der Ewigkeit von der Zeiterfahrung ausgeht. $\mathrm{Ob}$ man nun via negationis die Ewigkeit mit Augustin und der mystischen Tradition als Zeitlosigkeit oder ewiges $n u(n c)$ versteht, ob man nun gemäß der via analogiae mit Boethius Ewigkeit als die Gleichzeitigkeit aller Zeiten versteht, via causalitatis die Ewigkeit als die Bedingung der Möglichkeit von Zeitlichkeit oder aufgrund der via gloriae mit u.a. Swinburne Ewigkeit übersteigert als Endlosigkeit zumindest der Topologie wenn auch nicht der Metrik der Zeit; ${ }^{5}$ in all diesen Fällen handelt es sich um natürliche Theologien der Zeit, die von der Zeiterfahrung ausgehend spezifische kognitive Operationen vornehmen, um einen Ewigkeitsbegriff zu erhalten. Nicht einmal Karl Barth ist vor einer solchen gefeit. Zwar erkennt er die jeweils von ihm abgelehnten Ewigkeitsverständnisse als Ausdrücke menschlicher Übersteigerungsphantasien, aber die eigene Präferenz eines Ewigkeitsverständnisses als reiner oder freier Dauer wird nicht als Variation desselben Themas durchschaut. ${ }^{6}$ Auf diese Weise gibt es beinahe soviele Ewigkeitsverständnisse wie es Gotteslehren gibt. Und die Vielfalt und die Differenz der Ewigkeitsverständnisse ist in zweierlei Aspekten begründet: einmal in der spezifischen Beschreibung der Zeiterfahrung als Ausgangspunkt und einmal in der Wahl der jeweiligen kognitiven Operationen. Mögen diese alle auch logisch kohärent durchgefuihrt werden können und mögen auch innerhalb der entsprechenden Modelle die anderen Ewigkeitsverständnisse als defizitär ausgewiesen werden können, so wird doch deutlich, dass es sich letztlich um nichts anderes als kognitive Konstrukte handelt, die letztlich ein bestimmtes Verhältnis von Zeit und Ewigkeit absichern sollen. Damit aber sichern sie nicht nur bestimmte loci innerhalb von Theologien ab. Denn die Bestimmung des Zeit-Ewigkeitsverhältnisses ist viel mehr als nur ein Teilthema

\footnotetext{
$5 \mathrm{Zu}$ diesen unterschiedlichen Ewigkeitsauffassungen vgl. Markus Mühling, Liebesgeschichte Gott. Systematische Theologie im Konzept, Göttingen 2013, 215-234.

6 Vgl. Karl Barth, Kirchliche Dogmatik, Bd. II/I, Zürich ${ }^{4} 1958,688-693$.
} 
der Gotteslehre, der Eschatologie oder eines anderen locus. ZeitEwigkeitsverhältnisse entpuppen sich bei genauerem Hinsehen als umfassendes Strukturprinzip dogmatischer Systembildung - und zwar auch dort, wo dies nicht explizit von den Systemautoren erkannt werden mag. ${ }^{7}$

Man mag die Ursache für diesen Sachverhalt in hermeneutischlebensweltlichen Überlegungen zum Erfahrungsbegriff erblicken, aber vermutlich besteht die Ursache für die Mächtigkeit von Zeit-Ewigkeitsverhältnissen ebenso in einem Zug neuzeitlicher Systembildung an sich, der auch unter postmodernen Bedingungen versteckt weiter existiert: Wenn ein "modernes « System in Analogie zur Architektur ${ }^{8}$ durch die beiden Kennzeichen des organischen Zusammenhangs und der einheitlichen und geradlinigen Geschlossenheit ${ }^{9}$ charakterisiert werden kann, dann ist jede Systembildung selbst eine Ewigkeitskonstruktion: Jede Systembildung wird das Erscheinende im System repräsentativ abbilden müssen und damit in eine Statik überführen, die ungeachtet des Flusses allen Erscheinens "für immer" (systemrelativ) gilt. Auch wenn dieser Systembegriff in der Systematischen Theologie schon im 19. Jahrhundert als nicht wirklichkeitsresonierend durchschaut wurde,${ }^{10}$ so dass die meisten neuzeitlichen "Systematischen Theologien" in Wirklichkeit postsystematische Theologien sind, weil sie gerade auf Geschlossenheit verzichten müssen, so partizipieren sie doch häufig an dieser modernen Verewigung durch doppelte Inversion.

\footnotetext{
7 Dies lässt sich z.B. anhand des Systemaufbaus bei Albrecht Ritschl zeigen, vgl. Markus Mühling, Liebesgeschichte Gott, 235-241.

8 Systembildung kann auch insofern als Analogie zur Architektur verstanden werden, als Systembildungen durch Repräsentationen gekennzeichnet sind, deren Verbindungen durch Geraden zwischen Punkten veranschaulicht werden kann. Dies entspricht der gotischen Separation des Architekten vom Baumeister, die erst die Verwendung des Lineals und der Geraden durch Arbeitsteilung und leibliche Allotopie hervorgebracht hat.Vgl.Tim Ingold, Lines. A Brief History, London/New York 2007, 161, und David Turnbull, The ad hoc Collective Work of Building Gothic Cathedrals with Templates, String and Geometry, Science, Technology, and Human Value 18 (1993), 315-340.

9 Vgl. Immanuel Kant, Kritik der reinen Vernunft, 2. Aufl. 1787, Akademie Ausgabe, Bd. 3, Berlin 1905, B 708:»[D]ie Vernunfteinheit ist die Einheit des Systems. «In Kant, KrV AA, 538f., versteht Kant "unter einem System die Einheit der mannigfaltigen Erkenntnisse unter einer Idee».

${ }^{10} \mathrm{Vgl}$. zur Entwicklung des Systembegriffs Eilert Herms, Art. System, RGG ${ }^{4}$, Bd. 7, Tübingen 2004, 2007-2011.
} 


\section{Verewigung als (doppelte) Inversion in Moderne und Postmoderne}

In erfrischender und gewagter, ich denke aber treffender Weise, hat jüngst der Aberdeener Sozialanthropologe Tim Ingold Modernisierung als Inversion beschrieben. ${ }^{11}$ Diese besteht darin, dass das wahre Geschehen in den leeren Innenraum des Gebäudes repräsentativ verlegt und damit simplifiziert wird: Statt wahrer Pflanzen im Boden erscheinen Repräsentationen auf den Tapeten; statt der faltigen Struktur des wirklichen Bodens, der durch und in das Wetter diffundiert, erscheint die Oberfläche des Parketts, auf der Möbel als Inventar aufgestellt werden und, falls es um Dynamik geht, diese nur die gezähmte Dynamik des Verschiebens des Inventars ist. Der »kantische Reisende« betrachtet die Welt als geschlossen glatte Sphäre, deren Radius und Umfang durch die Formen der Anschauung also durch Raum und Zeit - gegeben sind, so dass Empirie nur in der Entdeckung der auf diesem glatten Boden verteilten individuellen Gegenstände bestehen kann. Jedes "moderne" System, das von der Geschlossenheit der Erkenntnisbedingungen ausgeht, hat damit nicht nur an einer repräsentativen Inversion des Außen ins Innen teil, sondern auch an derVerewigungstendenz des »Für-Immer«, das Neuheit nur als Variation von zeitlosen, "für immer" gültigen Themen und Strukturen zulässt. Da diese innersystematische Ewigkeit aber auf dem Repräsentationsgedanken beruht, kann sie natürlich nichts anderes als eine »Prothese der Ewigkeit ${ }^{12}$ sein.

Postmoderne Tendenzen mögen mit dem Gedanken des geschlossenen Systems und mit dem Gedanken der repräsentationalistischen Erkenntnis aufräumen. Aber eben indem sie aufräumen, partizipieren sie an der Struktur einer erneuten Inversion: Sie kehren das Innere nun buchstäblich erneut nach außen. Nicht mehr das geschlossene System ist nun federführend, sondern die Beschreibung allen Geschehens mit dramatischen Mitteln. Die Bühne des Theaters repräsentiert nicht mehr einfach die Wirklichkeit und führt sie der Prothese der Ewigkeit zu, sondern die Welt wird selbst als Theater und Bühne verstanden. Auf dieser kann zwar viel geschehen, aber wie zuvor alles an die vermeintlich basale, d.h. ewige Subjekt-Objekt-Trennung und die begriffliche Erfassung gebunden war, so erscheint nun alles als wechselnde Theaterrolle oder als Funktion, in der zur Not auch die Planken eines schwimmenden Schiffes zum Mast umgebaut

${ }^{11}$ Vgl.Tim Ingold, The Life of Lines, London/New York 2015, 69-72.

12 Der Ausdruck geht zurück auf Stanislaw Lem, Sterntagebücher, Frankfurt a.M.

${ }^{15} 1996,123$. 
werden können - und umgekehrt. ${ }^{13}$ An die Stelle der Begriffe sind die Rollen getreten, an die Stelle des grand narrative die vielfachen dezentralen Aufführungen in kleinen Theatern und auf den Straßen und an die Stelle der rein logischen Verknüpfung die Epik. An die Stelle der Verewigung im System bzw. im Geist oder Gott ist nun die Verewigung in den vielfältigen, kleinen, epischen Erzählungen getreten, die zwar inkommensurabel erscheinen mögen und bewusst auf Übersetzungen verzichten, die aber dennoch den Zuschauern nur als Variationen eines aufgeführten Themas erscheinen, wenn man auch nicht mehr sagen kann, warum sie das sind. Auch postmoderne Theoriebildungen leben so von Ewigkeitsprothesen und der Konstruktion des Junktims von "Für-immer-Dein«-Verheißungen und "Augenblick-verweile-doch«-Erwartungen.

\section{Wie spricht man von der Ewigkeit?}

\subsection{Gottes Ewigkeit}

Sind unsere Analysen richtig, dann befinden wir uns in einem Dilemma. Einerseits scheint jede Rede von Ewigkeit dem Funktionsmechanismus der »für-immer«-Versuchlichkeit zu unterliegen. Andererseits scheint aber gerade der Verzicht auf die Rede von Ewigkeit nicht zu gelingen, sondern nur in immer neue - und schlimmstenfalls undurchsichtige - Ewigkeitskonstruktionen zu führen. Diesen Zug teilt die Rede von der Ewigkeit freilich mit der Rede von Gott im Allgemeinen, zumal im Rahmen der Vorstellung von Gottes Einfachheit alle Wesenseigenschaften Gottes miteinander und mit der divinitas identisch sind - also auch die Ewigkeit. Und sollte man von Theologen nicht berechtigterweise einen Ausweg erwarten?

Ein solcher Ausweg soll im Folgenden angedacht werden. Will man nicht die beschriebenen Fehler einer negativen Theolgoie der Zeit machen, wird man anders vorgehen müssen. Man wird einerseits zu fragen haben, ob aufgrund der Selbstpräsentation Gottes Ewigkeit denkbar ist, und andererseits, ob und auf welche Weise der so gewonnene Ewigkeitsbegriff mit dem der Zeit zu vermitteln ist. Vorausgesetzt ist bei diesem Verfahren die Einsicht, dass Zeit und

${ }^{13}$ Vgl. Otto Neurath, Protokollsätze, Erkenntnis 3 (1932), 204-214, hier 208. Das Gleichnis wird von Neurath im antifundamentalistischen und holistischen Sinn schon seit 1913 genutzt. 
Ewigkeit ein Junktim oder ein Korrelat bilden, so dass das eine nicht ohne das andere zu haben ist.

Die Selbstpräsentation Gottes beruht auf der Beantwortung der Problemkreise: 1. Was ist das universe of discourse, wenn von "Gott" die Rede ist, d.h. was sind religionsphilosophische Minimalbedingungen der Rede von Gott? 2. Wie präsentiert sich Gott selbst? 3. Welche Folgerungen ergeben sich dabei für die Rede von der Ewigkeit Gottes?

1. Das "universe of discourse" möglicher Gottesbegriffe wird gewöhnlich durch eine Reihe religionsphilosophischer Bestimmungen angegeben, wie "das, worüber hinaus Größeres nicht denkbar ist ${ }^{14}{ }^{14}$, das Sein selbst ${ }^{15}$, »die alles bestimmende Wirklichkeit ${ }^{16}{ }^{16}$, das, woran der Mensch sein Herz gehängt bekommt «" ${ }^{17}$, the ultimate concern $\aleph^{18}$, oder $» d a s$, was die Zeit zusammenhält $\star^{19}$. Diese verschiedenen Definitionsversuche eines allgemeinen Gottesbegriffs schließen sich einerseits nicht notwendig aus, ergänzen sich andererseits aber auch nicht notwendig. Sie sind auch nicht traditionsfrei, sondern sind ja ihrerseits schon in unterschiedliche Theologien eingebettet. Ich möchte nun vorschlagen, keinen dieser Begriffe $\mathrm{zu}$ verwenden, sondern von einer eigenen Minimaldefinition auszugehen: Gott bezeichnet die dramatisch-narrative Integration aller stories. Diese Definition ist schon sehr voraussetzungsreich, daher seien ihre theologischen Hintergründe kurz erläutert. Unsere Wirklichkeit ist primär eine narrative, sie besteht aus stories oder Narrationen - und zwar auch unabhängig von menschlicher Erzähltätigkeit. Stories werden gelebt, bevor sie erzählt werden, wie MacIntyre sagt, und man müsste noch ergänzen: Stories geschehen, bevor sie gelebt werden. Das heißt wir leben in einer narrativen Welt. Dieser Narrationsbegriff ist also ein ontologischer Narrationsbegriff, und er enthält die Eigenschaften des Sich-Ereignens, der relationalen Verbundenheit von Ereignissen sowie der dynamischen Gerichtetheit von Ereignissen. Nicht vorausgesetzt sind Erzähler, Empfänger sowie bestimmte Relate oder Rollen in den Erzählungen. Vielmehr erscheinen diese

\footnotetext{
${ }^{14}$ Anselm von Canterbury, Proslogion (lat.-dt.), Stuttgart-Bad Cannstatt ${ }^{3} 1995,84$.

${ }^{15}$ Paul Tillich, Systematische Theologie I, Stuttgart ${ }^{6} 1980,273$.

${ }^{16}$ Rudolf Bultmann, Welchen Sinn hat es, von Gott zu reden?, in: GuV, Bd. I, hg. v. Michael Lattke, Tübingen 1961, 26-37; Wolfhart Pannenberg, Wissenschaftstheorie und Theologie, Frankfurt a.M. 1973, 304f.

${ }^{17}$ Die Formulierung in Luthers Großem Katechismus ist freilich aktivisch gewählt, vgl. Die Bekenntnisschriften der Evangelisch-lutherischen Kirche, herausgegeben im Gedenkjahr der Augsburgischen Konfession 1930, Göttingen ${ }^{9} 1982,560$.

${ }_{18}$ Paul Tillich, Systematic Theology I, Chicago 1967, 211-215.

${ }^{19}$ Robert W. Jenson, Systematic Theology I:The triune God, New York 1997, 54.
} 
erst durch intraaktive Differenzierungen dieser narrantologischen (von narrativ + ontologisch) Wirklichkeit. Diese narrantologische Wirklichkeit ist im Falle des Menschen immer mit dessen Wahrnehmung von ihr verbunden, die sich dann in der menschlichen Erzähltätigkeit widerspiegelt. Auf dieser abgeleiteten narratologischen nicht narrantologischen - Ebene gilt nun in der Tat, dass keine menschliche Erzählung jeweils die narrantologische Wirklichkeit repräsentieren kann. Narratologische Erzählungen beziehen sich zwar auf das Erscheinen der narrantologischen Wirklichkeit, indem sie mit dieser in Resonanz stehen, aber sie bilden sie nicht ab. Der Mensch nimmt also mit Hilfe unterschiedlicher Erzählungen, die sich nicht notwendig kohärent verhalten, die narrative Wirklichkeit zwar vermittelt durch diese partikularen Erzählungen wahr, aber doch unmittelbar, d.h. ohne Interpretationsakte. Dadurch wird seine personale und kulturelle Identität gebildet. Zu dieser gehören auch unterschiedliche Weglinienperspektiven, d.h. Weisen der Wahrnehmung der sich narrativ ereignenden Welt, in der Sein und Sollen noch ungeschieden sind bzw. in der es keine Trennung von Fakten und Werten gibt. Der christliche Glaube ist eine solche Weglinienperspektive oder eine Weise der Wahrnehmung, kein Glaubenssystem, kein (primär kognitives) Wirklichkeitsverständnis und kein Blickpunkt auf die Welt oder kein Standpunkt in der Welt. Weglinienperspektiven sind zwar nur narrativ und dynamisch beschreibbar, aber keine Weglinienperspektive kann je die dynamische Wirklichkeit als ganze beschreiben. Gleichwohl können mehrere mit ihr in harmonischer Resonanz stehen (oder in Dissonanz). Lyotards These vom Ende aller grand narratives ${ }^{20}$ wird hier durchaus als kritisches Prinzip wichtig, das vor Idolatrie schützen kann. Denn versteht man unter einem grand narrative im strengen Sinne die Integration aller stories auf der narrantologischen Ebene, dann kann es nur ein grand narrative geben, das sich niemals auf der narratologischen Ebene von partikularen Weglinienperspektiven aus nacherzählen lässt.

2. Wie präsentiert sich Gott selbst? Ist das zuvor Gesagte richtig, dann kann eine partikulare Weglinienperspektive eben nur dann positiv mit der sich ereignenden Wirklichkeit als ganzer resonieren, wenn Gott sich selbst erschließt, offenbart, oder präziser: präsentiert. Eine solche Selbstpräsentation Gottes wird notwendigerweise die geschaffene Struktur der Schöpfung nicht negieren können, d.h. sie wird selbst auf eine narrative-partikulare Weise geschehen, und zwar so, dass in der Verschränkung verschiedener narratologischer stories

${ }^{20}$ Vgl. Jean-Francois Lyotard, The Postmodern Condition. A Report on Knowledge, Manchester 1984, 9-16. 
eine Weglinienperspektive eröffnet wird, die positiv mit der narrantologischen Wirklichkeit resoniert: indem ein partikularer Mensch die eigene Lebensgeschichte in die Geschichte des Evangeliums eingezeichnet bekommt und vermittels dieser so entstehenden Weglinienperspektive die dynamische Wirklichkeit wahrnimmt. In diesem Einzeichnungs- und Wahrnehmungsgeschehen selbst, so die These, ereignet sich die göttliche Selbstpräsentation. Die Geschichte des Evangeliums, d.h. der frohen Botschaft, hat aber notwendig eine dreifache Struktur: 1. Im Handeln und Geschick Christi tritt der Anspruch auf, die verwirklichte basileia tou theou zu sein. 2. Diese basileia ist aber keine andere Herrschaft als die des Gottes Israels als Schöpfer der Welt, auf den sich Jesus personal als seinen Abba bezieht. 3. Die Krise und Ablehnung dieses Anspruchs Christi am Kreuz wird durch die Auferweckung Christi überwunden, die nicht durch natürliche Mittel wahrnehmbar ist, sondern nur durch die Gegenwart Gottes selbst in der Gemeinschaft der Glaubenden, d.h. durch den christuspräsentierenden Geist verbürgt ist. Diese Teilgeschichten bilden damit Identitätsbeschreibungen der Selbstpräsentation Gottes. Sie können abgekürzt werden durch die Namen »Vater, Sohn und Geist", die als solche keinen prädikativen Gehalt besitzen. ${ }^{21}$

3. Welche Folgerungen hat das narrative Selbstpräsentationsgeschehen für die Rede von der Ewigkeit? Eine Selbstpräsentation ist nur eine solche, wenn sie keine Lüge ist. Eine Selbstoffenbarung setzt vom Begriff her voraus, dass der Offenbarende in Wirklichkeit so ist, wie er erscheint. Präsentiert sich uns Gott auf der Weglinienperspektive der christlichen Praxis als dreieinige Geschichte, genauer als dreieinige Liebesgeschichte, dann muß er auch etsi mundus non daretur, ${ }^{22}$ als ob es keine Welt gäbe, eine dreieinige Liebesgeschichte sein; Gottes Wesen ist dann das einer dreieinigen Liebesgeschichte. Gott selbst ist ereignishaft, und diese Ereignishaftigkeit schließt nicht nur ein sequenzielles Geschehen und Narration mit ein, sondern es handelt sich auch, wie man ausführlicher zeigen könnte, ${ }^{23}$ um eine dramatische und nicht um eine epische Narration. Als solche schließt sie auch wahrhafte Neuheit und wahrhafte Kontingenz mit ein. Wichtig ist dabei, dass die drei Identitätsbeschreibungen Gottes

${ }^{21}$ Zur Theorie der starren Referenz von Namen vgl. Saul A. Kripke, Name und Notwendigkeit, Frankfurt a.M. 1993, 39f., und Hilary Putnam, Vernunft, Wahrheit und Geschichte, Frankfurt a.M. 1982, bes. 50-74.

${ }^{22}$ Zum Prinzip des etsi mundus non daretur als regulatives Prinzip der Theologie vgl. Markus Mühling, Resonances: Neurobiology, Evolution and Theology. Evolutionary Niche Construction, the Ecological Brain and Relational-Narrative Theology, Göttingen/Bristol, CT 2014, 19-21.

${ }^{23}$ Vgl. M. Mühling, Resonances, 123-128. 
durch eine logische Struktur gekennzeichnet sind: Die Geschichte des Vaters ist nicht spiegelbildlich auf die des Sohnes bezogen, und diese nicht spiegelbildlich auf die des Geistes; daher ist das Verhältnis zwischen ihnen asymmetrisch. Die Geschichte des Vaters zu kennen, erübrigt nicht, die Geschichte des Sohnes zu kennen, was nicht erübrigt, die Geschichte des Geistes zu kennen. Daher ist das Verhältnis der drei Identitätsbeschreibungen irreflexiv. Wenn aber die Geschichte des Geistes auf die des Sohnes bezogen ist, weil sie den Sohn vergegenwärtigt, und die Geschichte des Sohnes auf die des Vaters bezogen ist, dann ist auch die des Geistes auf die des Vaters bezogen. Es handelt sich also um ein transitives Verhältnis. Beziehungen, die asymmetrisch, irreflexiv und transitiv sind, heißen Ordnungsrelationen, denn sie ermöglichen die Individuation und Differenzierung. Alles und nur was in einer Ordnungsrelation steht, kann etwas Besonderes sein. Ordnungsrelationen erscheinen häufig: Die alphanumerische Reihenfolge eines Lexikons gehört genauso dazu wie die klassischen Modellierungen der immanenten Trinität, sei es nach östlicher oder westlicher Eigenart. Wenn das Axiom der Identität aller Eigenschaften Gottes miteinander und mit dem Wesen Gottes richtig ist, dann ist damit alles, was aufgrund der Basis der Selbstpräsentation Gottes über die Ewigkeit Gottes gedacht werden kann, gesagt: Ewigkeit ist die sequenzielle, Neuheit einschließende, trinitarische Liebesgeschichte, die zugleich eine Ordnungsrelation ist.

\subsection{Zeit zur Ewigkeit}

Wenn die Annahme, dass Zeit und Ewigkeit ein Junktim bilden, richtig ist, dann wird nun zu fragen sein, wie sich diese Ewigkeit zur Zeit verhält. Die Lösung besteht dabei darin, dass Ewigkeit und Zeit (und zwar Zeit in all ihren Dimensionen und Aspekten) darin übereinstimmen, dass sie eine Ordnungsrelation bilden und ebenso darin übereinstimmen, dass Zeit und Ewigkeit durch Ereignishaftigkeit und Narrativität gekennzeichnet sind - nicht umgekehrt: Raum und Zeit sind nicht der Rahmen, innerhalb derer sich Narrativität ereignet, sondern die Narrativität ist der Rahmen, der Räume und Zeiten entstehen lässt.

Dass Zeit in allen Dimensionen und Aspekten stets auch den Charakter einer Ordnungsrelation hat, dürfte unmittelbar einsichtig sein, denn die Relationen "vor" und "nach"sind genau solche Relationen.

Schwieriger zu begründen ist die Behauptung, die Zeit in all ihren Dimensionen und Aspekten sei durch narrative Ereignisse konstituiert. Denn dies gilt dann für die Dimension der physikalischen Zeit, 
der kulturellen Zeit, der phänomenalen Zeit und der ontischen Zeit. Es gilt ebenso für die unterschiedlichen Aspekte der Zeit zwischen Metrik und Topologie, Chronos und Kairos.

Die physikalische Zeit ist nicht, wie bei Newton oder Kant, die Form der Anschauung oder der unveränderliche Rahmen des mechanischen Geschehens, sondern zusammen mit dem Raum als Raumzeit konstituiert und abhängig vom Verhalten von Energiemasseäquivalenzen. Das Verhalten von Energiemasseäquivalenzen bringt also nach der speziellen und allgemeinen Relativitätstheorie die Zeit hervor, nicht umgekehrt. Zeit (und genauso Raum) sind also relational als Beziehungen zwischen Dingen zu verstehen. Als solche ist weder der Raum noch die Zeit isotrop, sondern beide sind anisotrop, d.h. sie können langsamer und schneller gedehnt oder gestreckt sein, etc. ${ }^{24}$

Die kulturelle Zeit ist ebenfalls duch die Ereignisse in ihr - genauer: durch die Art der narrativen Anordnung der Ereignisse - bestimmt: Kalender und Uhren sind Kulturgüter, Synchronie und Allochronie sozial-kommunikative Ordnungen. Wer sich dem entzieht, lebt nach dem modernen Selbstverständnis z.B. allochron "mittelalterlich". Unterschiedliche Kulturen haben unterschiedliche Kalender und unterschiedliche Kulturbereiche gestalten ihre Zeit nach unterschiedlichen Ereignishaftigkeiten. Ereignisse der Berufswelt mögen beispielsweise nicht zu denen der Familie, des Freundeskreises oder des Jahreskalenders passen. Durch das Erfordernis, simultan in mehreren kulturellen Zeitsequenzsystemen zu leben, entstehen Langweile und Zeitdruck sowie ein Großteil dessen, was Ricœur die "Aporien der Zeit $\aleph^{25}$ genannt hat.

Auch die phänomenale Zeit, die durch die Struktur der Wahrnehmung in Protention und Retention gegeben ist, ist keinesfalls gleichförmig durch die Erkenntnisbedingungen eines abstrakten Subjekts gegeben, sondern beruht auf der Leiblichkeit der Wahrnehmung. Da der Leib aber selbst keine statische, sondern eine dynamische und veränderbare Größe ist, ist die phänomenale Zeit durch die Ereignishaftigkeit und Narrativität des Leibes anisotrop strukturiert. Dabei ist es die Bewegungserfahrung des Leibes und eine Erfahrung von An- und Abwesenheit in Sequenzen, die die spezifische Gegenwart

\footnotetext{
${ }^{24}$ Eine allgemeinverständliche Einführung in die spezielle und allgemeine Relativitätstheorie bietet Albert Einstein, Über die spezielle und die allgemeine Relativitätstheorie, Berlin, Heidelberg ${ }^{23} 2001$.

${ }^{25}$ Einen kurzen Überblick über Ricœurs Aporien der Zeit gibt Verena Schlarb, Narrative Freiheit. Freiheit und Erzählen im Zusammenhang der Altenseelsorge, Leipzig 2015, 116-118.
} 
des Leibes als Mittelpunkt eines leiblichen, immer bewegten Koordinatensystems von Raum und Zeit, ein Präsenzfeld, bildet. ${ }^{26}$ Die Unmittelbarkeit der Leibwahrnehmung im Bewegungsgedächtnis impliziert daher nicht nur eine (nicht notwendig selbstbewusste) Gegenwartswahrnehmung, sondern immer auch ein protentionales Möglichkeitsfeld eines weiteren Bewegungsvollzugs, wie auch ein retentional leiblich erinnertes Von-Woher der Bewegung. In dem Maße nun, in dem die Bewegungen des Leibes passiv wie responsiv erfolgen, rhythmisch und arhythmisch, ist also auch das phänomenale Zeitfeld anisotrop.

Die Frage nach der ontischen Zeit ist die Frage nach der Zeit selbst. Schwierig bleibt überhaupt eine Antwort auf die Frage, ob und in welchem Rahmen diese Frage selbst sinnvoll ist. Im Rahmen postsystematischen Denkens wird man aber sagen können, dass die (abstrakte) ontische Zeit auf der Struktur von Sequenzhaftigkeit und damit Narrativität beruht, die im Werden Gottes selbst begründet ist und schöpfungstheologisch in der Narrativität der Welt ihre Resonanz findet.

Dass die Metrik der Zeit durch Ereignishaftigkeit konstituiert wird, wird schon in Aristoteles Zeitverständnis als Maß der Bewegung ${ }^{27}$ deutlich und konkretisiert sich in dem oben zur physikalischen Zeit Gesagten. Die Existenz einer Metrik der Zeit ist dabei an die Schöpfung gebunden. ${ }^{28}$

Da die Topologie selbst nichts anderes als das Vorher und Nachher von Ereignissen ist, ist per definitionem deutlich, dass auch die Topologie der Zeit von Ereignishaftigkeit abhängig ist. Die reine Topologie der Zeit - also abzüglich jeglicher Metrik - ist dabei aber weder isotrop noch anisotrop, weil Isotropie und Anisotropie immer ein $\mathrm{Ma} ß$ und eine Metrik erfordern.

Versteht man unter Chronologie der Zeit die Zeitfolge in der Verbindung von Topologie und Metrik sowie in der Verbindung von A-Reihe und B-Reihe, ist deutlich, dass die Chronologie ebenfalls narrativ-ereignishaft strukturiert ist.

Wichtiger ist aber, dass auch der Kairos selbst ereignishaft gebildet wird. Kairos meint hier nicht einfach einen Zeitpunkt und auch nicht einfach die rechte, erfüllte und ausgezeichnete Zeit, wie etwa

\footnotetext{
${ }^{26}$ Vgl. Maurice Merleau-Ponty, Phänomenologie der Wahrnehmung, Berlin 1966, 170, und dazu Yvonne Förster-Beuthan, Zeiterfahrung und Ontologie. Perspektiven moderner Zeitphilosophie, München 2012,170-177.

${ }^{27}$ Vgl. Aristoteles, Phys., 4,12.

${ }^{28}$ Vgl. Richard Swinburne, The Christian God, Oxford 1994, 75-80.
} 
bei Tillich, ${ }^{29}$ sondern bezieht sich auf die harmonische Resonanz verschiedener Zeiten: Besteht eine harmonische und nicht disharmonische Resonanz zwischen der Eigenbewegung eines Leibes und seiner Umwelt, erlebt der Leib einen Kairos im allgemeinen Sinne. ${ }^{30}$ Einen Kairos im allgemeinen Sinne erlebt der Leib auch, wenn es zur harmonischen Resonanz der Bewegung des Leibes in verschiedenen kulturellen Zeitsystemen kommt.

All diese verschiedenen Zeitdimensionen und Zeitaspekte der Welt sind also dadurch gekennzeichnet, dass sie auf narrativer Ereignishaftigkeit beruhen, dass sie eine Ordnungsrelation bilden, die meist zur Konstitution anisotroper Zeitfelder führt, die miteinander harmonisch oder disharmonisch resonieren können.

Man kann daher die geschaffene Zeit (oder genauer, die geschaffene Raumzeit) als eine geschaffene Resonanz zu Gottes Ewigkeit verstehen, die im Charakter der Ereignishaftigkeit und Strukturiertheit durch eine Ordnungsrelation eine Strukturgleichheit findet. Dabei gilt, dass nach der Sachordnung die Ewigkeit Gottes die ontische Bedingung der Möglichkeit für die geschaffene Zeit ist: Nicht weil die Welt zeitlich ist, ist von der Ewigkeit Gottes zu sprechen, sondern weil Gott in Ewigkeit ereignishaft im Werden ist, hat Gott in Resonanz zu seinem eigenen Werden eine zeitliche Welt geschaffen. Nach der Erkenntnisordnung gilt das Umgekehrte: Weil sich uns Gott narrativ in einer Verschränkung der Lebensgeschichten erschließt, die ihrerseits in den genannten Zeitdimensionen und -aspekten wahrgenommen wird, kann von der Ewigkeit Gottes gesprochen werden bzw. kann Gott als eine dramatisch-narrative Inklusion aller stories beschrieben werden.

\section{5. »Für-immer-Erzählungen « im Lichte der christlichen Weglinienperspektive}

\section{1 »Augenblick, verweile doch«?}

Im Rahmen des vorgestellten Denkens des Junktims von Ewigkeit und Zeit kann der Wunsch "Augenblick, verweile doch" unterschiedlich verstanden und bewertet werden, je nachdem, was man

\footnotetext{
${ }^{29}$ Vgl. Paul Tillich, Der Widerstreit von Raum und Zeit, Gesammelte Werke, Bd. 6, Stuttgart 1963, 10.

${ }^{30} \mathrm{Vgl}$.T. Ingold, Life of Lines, 71.
} 
unter "Augenblick " versteht:Versteht man unter "Augenblick" eine absolute Zeitlosigkeit im Sinne von Nicht-Ereignishaftigkeit, wäre der Wunsch gleichbedeutend mit dem Wunsch nach Nichtexistenz. Versteht man darunter eine ewige Gegenwart, die keine Retention und keine Bewegung in einen protentionalen Möglichkeitsraum einschließt, wäre der Wunsch nichts anderes als ein Zeugnis der Nichtanerkennung der Wirklichkeit - und damit Ausdruck einer ver-rückten oder sündhaften aktiven Beziehungsgestaltung in Respons auf alle passive Wahrnehmungserfahrung. Nur wenn man den "Augenblick" weder als Zeitlosigkeit, noch als abstrakte Grenze von Zukunft und Vergangenheit versteht, sondern als Ausdruck, dass sich die verschiedenen Erfahrungen von Zeit in den verschiedenen Dimensionen und Aspekten in harmonischer Resonanz befinden mögen, sich also kairotisch vollziehen, ohne Werden und Voranschreiten aufzuheben, könnte er positiv ein sinnvolles Bedürfnis menschlicher Geschöpflichkeit ausdrücken. In diesem Falle bestünde der diagnostizierte Mangel darin, dass zum einen jede geschöpfliche Retention nicht in einem bewussten Erinnern der gesamten vergangenen Geschichte besteht, also stets von einem Vergessen getragen ist (was unter geschöpflichen Bedingungen freilich auch sehr segensvoll sein kann), und dass zum anderen unsere narrativen Erfahrungen von Zeit sich höchstens punktuell kairotisch verhalten, sich also auf der narratologischen Ebene nicht inkludieren lassen. Es wäre daher der Wunsch nach einer narratologisch ausdrückbaren narrantologischen Integration der Geschichten in der Ewigkeit Gottes.

\subsection{Die Verheißung »Für immer Dein«}

Umgekehrt erscheinen dann auch alle innerweltlich ausgesprochenen "Für immer Dein"-Verheißungen als ver-rückt oder theologisch gesprochen sündhaft, weil sie entweder auf Selbstbetrug oder bewusstem Betrug beruhen müssen. Als Versprechungen von Zeitlosigkeit oder andauerndem Erleben einer spezifischen Gegenwartssequenz sind sie sinnlos; als Versprechungen der Integration der Zeit und eines kairotischen Zeiterlebens drücken sie im Bereich des Geschaffenen nichts anderes aus, als den hybriden Wunsch des Versprechenden, wie Gott sein zu wollen, oder zumindest die manipulativ versuchte Simulation der Prothese Ewigkeit. Wer sich aber an Prothesen und Simulationen klammert, wird die nichtsimulierte Wirklichkeit nicht mehr als solche erkennen können. Die Folge davon ist, dass die wahrhaftige Ewigkeitsverheißung als solche nicht anders als sub contrario erscheint, in der Kreuzeserfahrung der 
theologischen Epoché. Das korrekte »Für immer Dein«Versprechen hat daher auch immer den Doppelcharakter der Verheißung und Gerichtsankündigung, wie "retten" immer auch »richten" beinhaltet. ${ }^{31}$ Ausprechen kann dieses Versprechen nur die Ewigkeit in der Zeit selbst. "Für immer Dein«, das kann - wenn es keine Lüge sein soll - nur der inkarnierte Christus sagen bzw. Menschen an Christi statt kraft der Konkarnation des Geistes. Theologisch, also theoretisch, ist dieses Versprechen begrifflich relativ leicht zu beschreiben (d.h. die gesamte Dogmatik von Soteriologie bis zur Eschatologie anhand von Raum und Zeit zu entfalten). Was aber bedeutet das Versprechen für die Wahrnehmung des darauf vertrauenden Verheißungsempfängers?

\section{6. »Auch hat er die Ewigkeit in ihr Herz gelegt»}

Vor dem Hintergrund der hier vorgetragenen postsystematischen Überlegungen der christlichen Weglinienperspektive wird man zu einer bestimmten Interpretation der christlichen Erfahrung von Zeit und Ewigkeit sowie des christologischen "Für-immer-Dein«Versprechens kommen, das mithilfe einer Paraphrase des beliebten und oft auch arg strapazierten Textes Koh 3 ausgedrückt werden kann, insbesondere von Koh 3,11: "Auch hat er die Ewigkeit in ihr Herz gelegt«. Das Herz kann als Ausdruck der gesamten menschlichen Person, also als Ausdruck für das menschlich-partikulare Voneinander-und-Füreinander-Werden verstanden werden. Dieses vollzieht sich nicht einfach nur in der Zeit, sondern, traut man der christologischen "Für-immer-Dein"-Verheißung, stets auch in der Ewigkeit: Im Voneinander-und-Füreinander-Werden ist die Ewigkeit erfahrbar, eben weil sich die Zeit in der Ewigkeit vollzieht bzw. eine Resonanz der Ewigkeit darstellt. Erfahrbar ist diese Ewigkeit dabei in der Erfahrung der kairotischen Zeit im spezifischen Sinne, d.h. Erfahrung der harmonischen Resonanz aller geschaffenen Zeitdimensionen mit der Ewigkeit Gottes. Diese Erfahrung ist dann nichts anderes als seine eigenen narrativen, fragmentarischen $\mathrm{Ge}-$ schichten als in der Geschichte des Evangeliums wahrgenommen zu erfahren. ${ }^{32}$ Diese Wahrnehmungserfahrung ist nicht produzierbar,

\footnotetext{
${ }^{31}$ Vgl. Bernd Janowski, Der barmherzige Richter, in: Das Drama der Barmherzigkeit Gottes, hg. v. Ruth Scoralick, Stuttgart 2000, 33-91.

${ }^{32}$ Eine ausführliche Begründung zur vorgängigen Passivität dieser Wahrnehmung auf Grundlage der leiblichen Verfassung des Menschen, zu der immer auch das vorbewusste basale Selbst gehört, findet sich in Mühling, Resonances, 117-119.
} 
weil sie die Selbstpräsentation der Ewigkeit selbst voraussetzt. Geschieht dies, dann hat in der Tat »alles seine Zeit». Allerdings nicht im Sinne der üblichen Interpretation, nach der der Wechsel der Ereignisse mit ihrer lust- und leidhaften Qualität eben einfach als Gegebenheit hingenommen werden muss: "Alles hat seine Zeit» heißt dann, dass jedes Ereignis seine eigene Zeit bildet, unabhängig von anderen kulturellen Zeitkonstruktionen und letztlich unabhängig von jeder Metrik der Zeit: Die Ewigkeit Gottes hat eine Topologie, mitnichten aber eine Metrik. So gelesen wird "Alles hat seine Zeit" plötzlich utopisch und konkret zugleich. Utopisch, weil eben nicht die Alltagserfahrung des Wechsels von freud- und leidhaften Ereignissen gemeint ist, sondern dasjenige, metriklose Werden, in dem Ereignisse durch nichts als durch sich selbst konstituiert sind. Das ist in der Welt nicht der Fall, sondern gilt nur für die Ewigkeit Gottes. Wenn der Mensch daran vollständig partizipieren kann, dann nur in der eschatischen Hoffnung auf eine Theosis sola gratia, auf ein vollständiges Inkorporiertwerden in das beziehungshafte Werden der Ewigkeit Gottes. Zugleich ist "Alles hat seine Zeit" aber auch konkret: Nämlich dann wenn »seine» sich nicht nur auf »Alles», d.h. auf die Ereignisse bezieht, sondern auf die Verschränkung von Ewigkeit und Zeit im Inkarnierten. Dort wo mir alles, d.h. jedes einzelne denkbare Ereignis als ein Ereignis der Geschichte Christi erscheint, wo ich also Sequenzen meiner Narration als im Lichte der Narration Christi bewegt wahrnehme, nehme ich konkret die kairotische Verschränkung von Zeit in Ewigkeit wahr.

— Markus Mühling ist Professor für Systematische Theologie und Religionsphilosophie an der Leuphana Universität Lüneburg. Zu seinen Arbeitsgebieten gehören die Gotteslehre, die Eschatologie und das Gespräch von Theologie und Naturwissenschaften. 